\title{
Temporal Changes in Team Performance Indicators Which Differentiate Between Winning and Losing in Elite Gaelic Football
}

\author{
Declan Gamble $^{1,4}$, Andrew Mc Carren ${ }^{2}$, Jonathan Bradley ${ }^{3}$, Niall Moyna ${ }^{4}$ \\ ${ }^{1}$ Sport Northern Ireland Sports Institute, Ulster University, Newtownabbey, Northern Ireland \\ ${ }^{2}$ Insight Centre for Data Analytics, Dublin City University, Dublin, Ireland \\ ${ }^{3}$ Centre of Performance Analysis, Institute of Technology Carlow, Carlow, Ireland \\ ${ }^{4}$ School of Health and Human Performance, Dublin City University, Dublin, Ireland
}

Email address:

declangamble@sportni.net (D. Gamble)

\section{To cite this article:}

Declan Gamble, Andrew Mc Carren, Jonathan Bradley, Niall Moyna. Temporal Changes in Team Performance Indicators Which Differentiate Between Winning and Losing in Elite Gaelic Football. American Journal of Sports Science. Vol. 7, No. 4, 2019 , pp. $199-207$. doi: 10.11648/j.ajss.20190704.21

Received: November 18, 2019; Accepted: December 7, 2019; Published: December 19, 2019

\begin{abstract}
This study evaluated changes in performance indicators within winning and losing elite Gaelic football teams. Seven game statistics and 83 technical and tactical variables were measured during 24 games. Game statistics showed a significant decrease in ball in play time and increase in stoppage time between the first and second halves and between the first and fourth quarters. Significant differences between the first and second halves were evident in 10 variables ( 3 positive: 7 negative) in winning teams compared to 16 variables (4 positive: 12 negative) in losing teams. When the fourth quarter was compared to the first, significant differences were also found in 8 variables (negative) in winners and 23 variables (11 positive: 12 negative) in losers. Although certain technical and tactical variables improved in winners during halves and in losers during halves and quarters, when overall performance is evaluated across both match periods, decrements were more pronounced in both winners (15 vs. 3 ) and losers ( 24 vs. 15$)$. Both winners and losers experienced significant reductions in the frequency of team possession in the fourth quarter. Losers also demonstrated significant declines in total time in both team and individual player possession from the first to the second half and in passing (hand and kick) profiles across both halves and quarters. There was a significant increase in the percentage of successful free kick passes by winners in the second half and an increase in successful free kick passes and kick outs in losers in the fourth quarter. Both winners and losers reported significant reductions in turnover variables across halves and quarters. Defensive efficiency declined significantly in winners across match periods, which coincided with the significant increase in attacking efficiency of losers in the second half and fourth quarter. The attacking frequency declined significantly in winners in the second half and the fourth quarter and attacks originating in defense were also significantly lower in both winners and losers in the second half. Although both positive and negative differences were observed in the temporal changes exhibited by winners and losers across the match periods examined, the findings demonstrate that winners did not experience the same extant of technical and tactical performance decrement as losers, which partly explains the match outcomes associated with these games. In conclusion, knowledge of these temporal changes can be used to inform current preparation practices to enhance technical and tactical components and optimize match performance strategies.
\end{abstract}

Keywords: Team Sport, Performance Analysis, Football, Technical, Tactical

\section{Introduction}

Gaelic football is one of the native team sports played in Ireland. The popularity of this amateur sport is demonstrated by significant participation, volunteering, and attendance figures [1]. Nationally, Gaelic football attracts considerable television viewing audiences and media profile. Elite level competition is organized on an inter-county basis with the 
National Football League (NFL) preceding the All-Ireland Championship (AIC). Matches are played between two teams of 15 players (14 outfield players and a goalkeeper) on a rectangular pitch over two 35 min periods plus stoppage time. Once possession of the ball is gained, players can retain possession by passing with either hand or foot and scores are achieved by kicking or hand fisting the ball over the bar between the posts ( 1 point) or by kicking the ball below the crossbar for a goal (3 points).

Match play is often characterized by rapid transitions from defense to offence as teams attack and try to score in their opposition's defensive zone [2]. However, tactics adopted by modern Gaelic football teams, often involves withdrawing some or all of their attacking players to create a defensive screen $45-65 \mathrm{~m}$ from their goal [2]. This replicates the evolving compact formations observed in other team sports such as soccer, Australian football and rugby, which encompass the concentration of defensive actions in narrow bands around the central regions of the pitch [3]. This strategy enables teams to close space by increasing player density [3], and as a consequence this can negate the dynamic flow of the game by limiting the available space for attacking teams to exploit. Nevertheless, evaluation of the technical and tactical performance indicators that contribute to effective offence and defense and overall outcome [4], is important for coaches as this information can inform their preparation and assist with optimizing performance strategies that counteract the tactical deployment of opposition players.

Gaelic football coaches and practitioners have traditionally relied on generic literature from the other football codes [5], due to the lack of studies involving Gaelic games. However, emerging research in Gaelic football is beginning to address the current knowledge gaps and a few studies have documented technical performance indicators relating to; counterattacking [6], kick out distribution [7], comparing teams competing at different levels $[8,9]$, or era's [10], and winning $[2,11]$. Although there are currently no studies in Gaelic football that have examined differences in technical performance across match periods, previous investigations have reported decrements in player physical performance profiles between match halves and specific intervals of 15 $17.5 \mathrm{~min}$ [12-14]. In soccer, a significant decline in physical (total distance and high intensity running with and without the ball) and technical (involvements with the ball, short passes and successful short passes) performance was demonstrated between the first and second halves of games [15]. Similarly, running performance was reported to decline in soccer players between match halves and across $15 \mathrm{~min}$ intervals although no significant differences were found in skill-related performance variables between these match periods [16]. In contrast, in rugby league players, decrements in physical performance (distance travelled and number of collisions) observed in the final stages of matches were associated with significant declines in skill rating and number of skill involvements [17]. Though decrements in physical performance of players occur in the latter stages of matches, the influence and association of these decrements on skill related variables needs further investigation.

While the team performance indicators associated with winning Gaelic football games have already been elucidated [2, 11] examination of technical performance across match periods could enhance current understanding and provide coaches and practitioners with further insights relating to the preparation strategies required to win games. The aim of this investigation was to evaluate temporal changes in technical performance indicators between the first and second halves and from the first to the fourth quarter in winning and losing Gaelic football teams.

\section{Methods}

\subsection{Match Sample}

The technical and tactical performance indicators from 13 elite Gaelic football teams were examined during 14 intercounty Division 1 NFL and 10 AIC games. This match sample included 1 semi-final and final from the NFL and 2 semi-finals and finals from the AIC. The team ratings were determined using the Elo rating system for Gaelic football [18]. Both playing time and stoppage time was included in the total duration of each half. Quarters were calculated by dividing each half by 2 . For example a first half lasting 36 min, resulted in quarter 1 and quarter 2 being $18 \mathrm{~min}$ in duration, whereas a second half lasting $38 \mathrm{~min}$ led to quarter 3 and quarter 4 being 19 min in duration.

\subsection{Experimental Procedures and Operational Definitions}

The experimental procedures and operational definitions used in this study have been described in detail previously [2]. Match footage from internal team video recordings and from external media broadcasters was imported and coded using a custom built tagging panel in Dartfish (v8) TeamPro software (Fribourg, Switzerland). Following data validation, the coding events were then exported into Microsoft Excel (Microsoft, USA) and transformed and collated for specific match periods to facilitate statistical analysis.

\subsection{Reliability Assessment}

Intra-rater reliability was determined from 2 games randomly selected and coded twice over a 4-week period. The lowest intraclass correlation coefficient (ICC) recorded for halves was 0.90 (attack origin defense), whereas the lowest score for quarters was 0.89 (attack origin attack). All other variables had an ICC $>0.90$ (mean 0.98), demonstrating excellent reliability [19].

\subsection{Statistical Analysis}

The relative differences in game statistics and performance indicators between winners and losers were analyzed across match halves and between quarter 1 and quarter 4 using SPSS for Windows (Version 24; SPSS Inc., Chicago, USA) with statistical significance accepted at $p \leq 0.05$. Differences between winners and losers that were found to be normally distributed using the Shapiro-Wilk test were analyzed using a 
one-sample t-test. Differences which did not reflect a normal distribution, were examined using a Wilcoxon signed rank test. Descriptive statistics are presented as mean \pm SD.

\section{Results}

\subsection{Match Characteristics}

There were no significant differences demonstrated in the Elo ratings of winners $(1822.1 \pm 184.6)$ compared to losers $(1753.6$ \pm 174.6 ). In 14 of the 24 games the margin of victory was $\geq 6$ points, and the difference in the remaining 10 games was $\leq 5$ points. With respect to match periods, there was no significant difference in average playing time (mean $\pm \mathrm{SD}$ ) between the first and second halves (36:50 \pm 0:53 vs. 37:21 $\pm 1: 11 \mathrm{~min}: \mathrm{sec})$ or between the first and fourth quarters (18:25 \pm 0:26 vs. 18:40 \pm 0:35 min:sec), respectively. However, there was a significant decrease in ball in play times from the first to the second half
$(19: 16 \pm 2: 10$ vs. $17: 52 \pm 1: 51 \mathrm{~min}: \mathrm{sec})$ and from the first to the fourth quarter $(9: 31 \pm 1: 11$ vs. 8:36 $\pm 1: 21 \mathrm{~min}: \mathrm{sec})$. This was associated with a significant increase in stoppage time across both halves (17:34 $\pm 2: 30$ vs. 19:29 $\pm 2: 31 \mathrm{~min}: \mathrm{sec})$ and quarters (8:54 $\pm 1: 18$ vs. 10:04 $\pm 1: 39$ min:sec). Furthermore, there was a significant increase in the number of substitutions made in the second half and in the fourth quarter in both winners and losers (Table 1). There was a significant increase in the number of yellow cards issued to winners in both the second half and the fourth quarter but not in losers. The number of black cards issued to winners and losers was also significantly higher in the second half and only in losers in the fourth quarter. There was no significant differences in the number of red cards received between halves or quarters in winners or losers. The main insights from the results are summarized below according to specific aspects of game play.

Table 1. Summary of comparisons in match statistics for match halves and quarters, winners and losers, mean \pm SD.

\begin{tabular}{|c|c|c|c|c|c|c|c|c|}
\hline Period & Half & & & & Quarter & & & \\
\hline 24 Games & Winners & & Losers & & Winners & & Losers & \\
\hline Variable & First & Second & First & Second & First & Fourth & First & Fourth \\
\hline Substitutions (n) & $0.3 \pm 0.6$ & $4.7 \pm 1.2^{\beta}$ & $0.7 \pm 0.9$ & $4.8 \pm 1.1^{\beta}$ & $0.2 \pm 0.5$ & $3.2 \pm 1.1^{\alpha}$ & $0.2 \pm 0.4$ & $2.5 \pm 1.0^{\beta}$ \\
\hline Yellow cards (n) & $0.6 \pm 0.6$ & $1.0 \pm 1.0^{\beta}$ & $0.6 \pm 0.8$ & $0.6 \pm 0.8$ & $0.2 \pm 0.4$ & $0.8 \pm 0.9^{\beta}$ & $0.3 \pm 0.5$ & $0.4 \pm 0.8$ \\
\hline Black cards (n) & $0.0 \pm 0.2$ & $0.3 \pm 0.6^{\beta}$ & $0.1 \pm 0.3$ & $0.5 \pm 0.6^{\beta}$ & $0.0 \pm 0.2$ & $0.3 \pm 0.5$ & $0.1 \pm 0.3$ & $0.4 \pm 0.6^{\beta}$ \\
\hline Red card / BCNR (n) & $0.0 \pm 0.2$ & $0.1 \pm 0.3$ & $0.0 \pm 0.2$ & $0.2 \pm 0.4$ & $0.0 \pm 0.0$ & $0.1 \pm 0.3$ & $0.0 \pm 0.0$ & $0.1 \pm 0.3$ \\
\hline
\end{tabular}

BCNR $=$ Black card not replaced. Symbols indicate significantly different $(p \leq 0.05)$ from either first half or first quarter, using either a one sample t-test $(\alpha)$ or Wilcoxon signed rank test $(\beta)$.

\subsection{Possession}

There was no significant difference in any possession variables between the first and second halves in winners. However, in the second half, the total duration of both team and individual player possession was significantly reduced in losers. This was associated with a significant decline in team possessions originating in both defense and midfield. In addition, the total number of player possessions and player possessions originating in midfield also decreased in losers in the second half. Both winners and losers demonstrated a significant decline in team possession in the fourth quarter and this coincided with a decline in team possessions originating in defense in losers. In the fourth quarter, the total number of player possessions decreased significantly in losers, manifesting in a significant decline in player possessions originating in defense and midfield, in contrast to the decline in player possessions originating in attack experienced by winners.

Table 2. Summary of univariate comparisons for match halves and quarters, winners and losers - possession, mean $\pm S D$.

\begin{tabular}{|c|c|c|c|c|c|c|c|c|}
\hline \multirow{3}{*}{$\begin{array}{l}\text { Period } \\
24 \text { Games } \\
\text { Variable }\end{array}$} & \multicolumn{4}{|l|}{ Half } & \multicolumn{4}{|l|}{ Quarter } \\
\hline & \multicolumn{2}{|l|}{ Winners } & \multicolumn{2}{|l|}{ Losers } & \multicolumn{2}{|l|}{ Winners } & \multicolumn{2}{|l|}{ Losers } \\
\hline & First & Second & First & Second & First & Fourth & First & Fourth \\
\hline Team possession (n) & $36.5 \pm 5.2$ & $35.1 \pm 4.2$ & $36.3 \pm 4.8$ & $35.5 \pm 5.1$ & $19.2 \pm 2.9$ & $17.0 \pm 2.0^{\alpha}$ & $18.9 \pm 2.8$ & $17.0 \pm 3.1^{\alpha}$ \\
\hline TM possession $(\%)$ & $49.4 \pm 8.7$ & $52.9 \pm 7.6$ & $50.6 \pm 8.7$ & $47.1 \pm 7.6$ & $49.8 \pm 9.4$ & $53.6 \pm 9.9$ & $50.2 \pm 9.4$ & $46.4 \pm 9.9$ \\
\hline TM possession (s) & $485.4 \pm 83.0$ & $488.6 \pm 98.2$ & $504.9 \pm 124.0$ & $431.3 \pm 76.8^{\alpha}$ & $239.1 \pm 45.8$ & $239.9 \pm 65.4$ & $245.5 \pm 68.3$ & $206.2 \pm 53.8$ \\
\hline TM possession av. (s) & $13.4 \pm 2.2$ & $14.2 \pm 3.6$ & $14.3 \pm 4.6$ & $12.4 \pm 2.8$ & $12.6 \pm 2.5$ & $14.3 \pm 4.3$ & $13.2 \pm 4.1$ & $12.4 \pm 3.4$ \\
\hline TM poss. origin MF (n) & $12.6 \pm 3.8$ & $10.3 \pm 3.6$ & $10.2 \pm 4.2$ & $11.6 \pm 4.4^{\beta}$ & $7.1 \pm 2.7$ & $4.7 \pm 2.3$ & $5.5 \pm 3.5$ & $5.6 \pm 2.4$ \\
\hline TM poss. origin AT (n) & $4.2 \pm 2.3$ & $4.2 \pm 2.2$ & $3.8 \pm 1.4$ & $4.0 \pm 2.4$ & $2.0 \pm 1.5$ & $2.1 \pm 1.2$ & $2.0 \pm 1.0$ & $2.3 \pm 1.9$ \\
\hline Player possession (n) & $149.8 \pm 21.9$ & $149.0 \pm 31.9$ & $158.1 \pm 32.7$ & $139.2 \pm 21.3^{\alpha}$ & $76.3 \pm 13.1$ & $72.7 \pm 18.9$ & $79.4 \pm 20.0$ & $67.3 \pm 14.3^{\alpha}$ \\
\hline PL possession (s) & $320.6 \pm 69.8$ & $315.6 \pm 66.5$ & $329.5 \pm 96.5$ & $277.4 \pm 59.9^{\alpha}$ & $153.4 \pm 33.2$ & $150.5 \pm 41.0$ & $159.6 \pm 52.2$ & $129.1 \pm 38.2$ \\
\hline PL possession av. (s) & $2.1 \pm 0.3$ & $2.1 \pm 0.3$ & $2.1 \pm 0.3$ & $2.0 \pm 0.3$ & $2.0 \pm 0.2$ & $2.1 \pm 0.3$ & $2.0 \pm 0.4$ & $1.9 \pm 0.3$ \\
\hline PL poss. origin DF (n) & $40.1 \pm 10.8$ & $41.3 \pm 8.2$ & $45.1 \pm 12.3$ & $39.6 \pm 13.3$ & $20.9 \pm 7.0$ & $20.6 \pm 6.3$ & $22.6 \pm 7.9$ & $17.9 \pm 6.9^{\alpha}$ \\
\hline PL poss. origin AT (n) & $37.3 \pm 8.8$ & $33.3 \pm 11.3$ & $32.2 \pm 10.7$ & $36.2 \pm 13.5$ & $19.6 \pm 5.9$ & $15.4 \pm 6.3^{\alpha}$ & $16.4 \pm 5.9$ & $18.5 \pm 7.9$ \\
\hline
\end{tabular}

$\mathrm{TM}=$ team, Poss. = possession, av. = average, $\mathrm{DF}=$ defense, $\mathrm{MF}=$ midfield, $\mathrm{AT}=$ attack, $\mathrm{PL}=$ player. Symbols indicate significantly different $(p \leq 0.05)$ from either first half or first quarter, using either a one sample t-test $(\alpha)$ or Wilcoxon signed rank test $(\beta)$. 


\subsection{Passing}

There was no significant difference in the passing characteristics between any match periods in winners (Table 3). However, there was a significant reduction in the total number of combined hand and kick passes executed by losers across both halves and quarters, resulting from the significant decline in the number of hand and kick passes performed. In losers, there was also a significant reduction in the number of successful combined passes in the second half reflected largely in the significant decrease in the number of successful hand passes performed in both the second half and in the fourth quarter. Similarly, the number of unsuccessful combined passes declined significantly in both the second half and fourth quarter in losers. This coincided with a significant decrease in the number of unsuccessful kick passes performed by losers in the fourth quarter, which was reflected in the significant increase in the percentage success of kick passes in this period.

Table 3. Summary of univariate comparisons for match halves and quarters, winners and losers - passing, mean $\pm S D$.

\begin{tabular}{|c|c|c|c|c|c|c|c|c|}
\hline Period & Half & & & & Quarter & & & \\
\hline 24 Games & Winners & & Losers & & Winners & & Losers & \\
\hline Variable & First & Second & First & Second & First & Fourth & First & Fourth \\
\hline Hand+kick pass (n) & $123.8 \pm 20.4$ & $123.9 \pm 31.6$ & $134.7 \pm 32.0$ & $114.0 \pm 20.5^{\alpha}$ & $63.5 \pm 12.3$ & $60.3 \pm 17.9$ & $67.2 \pm 18.7$ & $54.8 \pm 13.7^{\alpha}$ \\
\hline HKP success (n) & $113.0 \pm 19.0$ & $114.0 \pm 32.6$ & $122.5 \pm 34.0$ & $104.0 \pm 21.5^{\alpha}$ & $57.3 \pm 12.1$ & $55.4 \pm 18.5$ & $60.7 \pm 19.9$ & $50.5 \pm 14.0$ \\
\hline HKP success (\%) & $91.4 \pm 2.6$ & $91.3 \pm 4.4$ & $90.1 \pm 4.8$ & $90.9 \pm 4.1$ & $90.2 \pm 5.0$ & $91.0 \pm 5.0$ & $89.2 \pm 5.6$ & $91.6 \pm 5.0$ \\
\hline HKP unsuccess. (n) & $10.7 \pm 3.5$ & $9.9 \pm 3.6$ & $12.2 \pm 4.1$ & $9.9 \pm 3.9^{\alpha}$ & $6.2 \pm 3.0$ & $4.9 \pm 2.2$ & $6.5 \pm 2.6$ & $4.3 \pm 2.5^{\alpha}$ \\
\hline Hand pass (n) & $83.3 \pm 17.3$ & $85.4 \pm 30.3$ & $93.9 \pm 31.9$ & $76.3 \pm 19.8^{\alpha}$ & $42.3 \pm 11.0$ & $41.6 \pm 16.3$ & $46.9 \pm 18.9$ & $37.0 \pm 12.0^{\circ}$ \\
\hline HP success (n) & $81.1 \pm 16.8$ & $83.5 \pm 29.6$ & $91.0 \pm 31.8$ & $74.3 \pm 19.9^{\alpha}$ & $40.9 \pm 10.7$ & $40.5 \pm 16.1$ & $45.5 \pm 18.5$ & $35.9 \pm 11.9^{\alpha}$ \\
\hline HP success (\%) & $97.5 \pm 1.8$ & $97.8 \pm 1.7$ & $96.6 \pm 1.9$ & $97.0 \pm 2.5$ & $96.9 \pm 2.9$ & $96.8 \pm 4.1$ & $97.3 \pm 2.0$ & $97.1 \pm 3.8$ \\
\hline HP unsuccess. (n) & $2.1 \pm 1.5$ & $1.9 \pm 1.4$ & $2.8 \pm 1.4$ & $2.1 \pm 1.5$ & $1.3 \pm 1.1$ & $1.2 \pm 1.1$ & $1.3 \pm 1.0$ & $1.0 \pm 1.2$ \\
\hline HP unsuccess. (\%) & $2.5 \pm 1.8$ & $2.2 \pm 1.7$ & $3.4 \pm 1.9$ & $3.0 \pm 2.5$ & $3.2 \pm 2.9$ & $3.2 \pm 4.1$ & $2.8 \pm 2.0$ & $2.9 \pm 3.8$ \\
\hline Kick pass (n) & $40.5 \pm 9.7$ & $38.5 \pm 5.8$ & $40.8 \pm 7.7$ & $37.6 \pm 7.9^{\alpha}$ & $21.2 \pm 6.0$ & $18.6 \pm 3.9$ & $20.3 \pm 5.0$ & $17.8 \pm 4.7^{\alpha}$ \\
\hline KP success $(\%)$ & $78.5 \pm 6.5$ & $78.8 \pm 8.8$ & $76.7 \pm 9.7$ & $79.4 \pm 7.4$ & $77.1 \pm 9.9$ & $79.0 \pm 11.8$ & $73.9 \pm 14.5$ & $82.1 \pm 10.5^{\circ}$ \\
\hline KP unsuccess. (n) & $8.6 \pm 3.1$ & $8.0 \pm 3.4$ & $9.4 \pm 4.1$ & $7.8 \pm 3.6$ & $4.8 \pm 2.6$ & $3.7 \pm 1.9$ & $5.2 \pm 3.0$ & $3.3 \pm 2.3^{\alpha}$ \\
\hline KP unsuccess. (\%) & $21.5 \pm 6.5$ & $21.2 \pm 8.8$ & $23.3 \pm 9.7$ & $20.7 \pm 7.4$ & $22.9 \pm 9.9$ & $21.0 \pm 11.8$ & $26.1 \pm 14.5$ & $17.9 \pm 10.5^{\circ}$ \\
\hline
\end{tabular}

$\mathrm{HKP}=$ Hand+Kick pass, unsuccess. $=$ unsuccessful, $\mathrm{HP}=$ hand pass, $\mathrm{KP}=$ kick pass. Symbols indicate significantly different $(p \leq 0.05)$ from either first half or first quarter, using either a one sample t-test $(\alpha)$ or Wilcoxon signed rank test $(\beta)$.

\subsection{Dead Ball Distribution}

In the second half, winners had a significant reduction in the number of unsuccessful dead ball free kick passes, which was reflected in a significant increase in their percentage success rate (Table 4). Additionally, winners had a significant increase in the number of unsuccessful dead ball kick outs executed in the second half. In contrast, losers had a significant reduction in the number of unsuccessful dead ball kick outs and kick passes in the fourth quarter and this resulted in a significant increase in their percentage dead ball kick out and kick pass success.

Table 4. Summary of univariate comparisons for match halves and quarters, winners and losers - dead ball distribution, mean \pm SD.

\begin{tabular}{|c|c|c|c|c|c|c|c|c|}
\hline \multirow{3}{*}{\begin{tabular}{|l} 
Period \\
24 Games \\
Variable \\
\end{tabular}} & \multicolumn{4}{|l|}{ Half } & \multicolumn{4}{|l|}{ Quarter } \\
\hline & Winners & & Losers & & Winners & & Losers & \\
\hline & First & Second & First & Second & First & Fourth & First & Fourth \\
\hline Dead ball (n) & $21.3 \pm 4.5$ & $21.4 \pm 4.3$ & $22.9 \pm 4.7$ & $23.9 \pm 4.1$ & $11.2 \pm 2.6$ & $11.0 \pm 2.6$ & $11.7 \pm 2.6$ & $11.6 \pm 2.9$ \\
\hline DBKP success (n) & $14.3 \pm 3.2$ & $13.8 \pm 3.0$ & $14.7 \pm 4.7$ & $16.4 \pm 3.2$ & $7.1 \pm 2.0$ & $7.0 \pm 2.1$ & $7.2 \pm 3.0$ & $8.2 \pm 2.3$ \\
\hline DBKP success (\%) & $80.2 \pm 8.1$ & $77.8 \pm 10.6$ & $73.8 \pm 12.9$ & $80.8 \pm 11.9$ & $76.7 \pm 14$ & $77.8 \pm 15.4$ & $71.9 \pm 19.1$ & $85.5 \pm 14.5^{\circ}$ \\
\hline DBKP unsuccess. (n) & $3.6 \pm 1.9$ & $4.3 \pm 2.3$ & $5.1 \pm 2.6$ & $4.2 \pm 2.8$ & $2.3 \pm 1.6$ & $2.1 \pm 1.5$ & $2.8 \pm 1.8$ & $1.5 \pm 1.5^{\alpha}$ \\
\hline DBKP unsuccess. (\%) & $19.8 \pm 8.1$ & $22.2 \pm 10.6$ & $26.2 \pm 12.9$ & $19.2 \pm 11.9$ & $23.3 \pm 14.0$ & $22.2 \pm 15.4$ & $28.1 \pm 19.1$ & $14.6 \pm 14.5^{\circ}$ \\
\hline DBFKP success (n) & $6.3 \pm 3.1$ & $6.3 \pm 2.4$ & $6.0 \pm 3.7$ & $6.8 \pm 3.1$ & $2.9 \pm 1.6$ & $3.2 \pm 1.2$ & $2.9 \pm 2.5$ & $3.4 \pm 2.1$ \\
\hline DBFKP success (\%) & $91.4 \pm 9.4$ & $97.3 \pm 6.5^{\alpha}$ & $94 \pm 11.2$ & $96.1 \pm 6.9$ & $89.8 \pm 15.1$ & $96.9 \pm 11.2$ & $96.4 \pm 12$ & $95.0 \pm 10.2$ \\
\hline DBFKP unsuccess. (n) & $0.6 \pm 0.6$ & $0.2 \pm 0.5^{\beta}$ & $0.4 \pm 0.6$ & $0.5 \pm 0.8$ & $0.3 \pm 0.5$ & $0.1 \pm 0.3$ & $0.1 \pm 0.3$ & $0.3 \pm 0.5$ \\
\hline DBFKP unsuccess. (\%) & $8.6 \pm 9.4$ & $2.7 \pm 6.5^{\alpha}$ & $6.0 \pm 11.2$ & $3.9 \pm 6.9$ & $10.2 \pm 15.1$ & $3.1 \pm 11.2$ & $3.6 \pm 12.0$ & $5.0 \pm 10.2$ \\
\hline Dead ball kick out (n) & $9.5 \pm 2.0$ & $10.8 \pm 3.2$ & $12.0 \pm 2.2$ & $12.0 \pm 3.0$ & $5.2 \pm 2.0$ & $5.5 \pm 2.0$ & $6.2 \pm 1.7$ & $5.5 \pm 2.5$ \\
\hline DBKO success (n) & $6.6 \pm 1.4$ & $6.8 \pm 2.4$ & $7.4 \pm 1.7$ & $8.5 \pm 3.0$ & $3.3 \pm 1.3$ & $3.4 \pm 1.7$ & $3.6 \pm 1.2$ & $4.4 \pm 2.1$ \\
\hline DBKO success (\%) & $71.2 \pm 16.0$ & $65.2 \pm 18.1$ & $62.8 \pm 15.9$ & $71.3 \pm 20.2$ & $68.3 \pm 22.8$ & $65.3 \pm 22.1$ & $61.5 \pm 22.7$ & $83.3 \pm 20.2^{\circ}$ \\
\hline DBKO unsuccess. (\%) & $28.8 \pm 16.0$ & $34.8 \pm 18.1$ & $37.2 \pm 15.9$ & $28.7 \pm 20.2$ & $31.7 \pm 22.8$ & $34.7 \pm 22.1$ & $38.5 \pm 22.7$ & $16.7 \pm 20.2^{\circ}$ \\
\hline
\end{tabular}

$\mathrm{DBKP}=$ dead ball kick pass, unsuccess. = unsuccessful, DBFKP = dead ball free kick pass, DBKO = dead ball kick out. Symbols indicate significantly different $(p \leq 0.05)$ from either first half or first quarter, using either a one sample t-test $(\alpha)$ or Wilcoxon signed rank test $(\beta)$. 


\subsection{Offence}

Among winners, the number of attacks was significantly lower in both the second half and fourth quarter. In addition, the number of attacks originating in midfield was significantly lower in winners in both the second half and fourth quarter, whereas the number of attacks originating in defence in the second half was significantly lower in losers. Losers had a significant increase in the number of shots in the second half, resulting in a significantly enhanced attacking efficiency in both the second half and the fourth quarter.

\subsection{Defense}

Winners had a significantly lower number of turnovers in the fourth quarter. The number of turnovers gained in midfield declined significantly in winners in the second half and fourth quarter, whereas the number of turnovers gained in defense decreased significantly in losers in both the second half and fourth quarter. The percentage of successful tackles executed by winners was also significantly lower in the second half, coinciding with a significant reduction in the number of tackles originating in attack in the fourth quarter. The overall defensive efficiency in winners was significantly lower in both the second half and fourth quarter. In losers, the total number of combined defensive actions and number of defensive actions occurring in defense was significantly lower in the fourth quarter.

Table 5. Summary of univariate comparisons for match halves and quarters, winners and losers - offensive play, mean \pm SD.

\begin{tabular}{|c|c|c|c|c|c|c|c|c|}
\hline \multirow{3}{*}{$\begin{array}{l}\text { Period } \\
24 \text { Games } \\
\text { Variable }\end{array}$} & \multicolumn{4}{|l|}{ Half } & \multicolumn{4}{|l|}{ Quarter } \\
\hline & Winners & & Losers & & Winners & & Losers & \\
\hline & First & Second & First & Second & First & Fourth & First & Fourth \\
\hline Attack (n) & $22.1 \pm 3.9$ & $19.3 \pm 4.1^{\alpha}$ & $19.1 \pm 3.3$ & $19.3 \pm 3.3$ & $11.3 \pm 2.6$ & $8.3 \pm 2.7^{\alpha}$ & $9.9 \pm 2.5$ & $9.0 \pm 1.8$ \\
\hline AT origin DF (n) & $11.1 \pm 2.9$ & $10.5 \pm 2.7$ & $11.0 \pm 3.4$ & $9.3 \pm 3.4^{\alpha}$ & $5.1 \pm 1.7$ & $4.6 \pm 2.2$ & $5.5 \pm 2.2$ & $4.5 \pm 2.0$ \\
\hline AT origin MF (n) & $10.2 \pm 3.4$ & $7.9 \pm 3.0^{\alpha}$ & $7.6 \pm 3.1$ & $9.0 \pm 3.1$ & $5.8 \pm 2.4$ & $3.4 \pm 1.7^{\alpha}$ & $4.1 \pm 2.8$ & $4.1 \pm 1.7$ \\
\hline AT origin AT (n) & $0.9 \pm 0.9$ & $0.9 \pm 1.1$ & $0.5 \pm 0.6$ & $0.9 \pm 1.1$ & $0.4 \pm 0.7$ & $0.3 \pm 0.4$ & $0.3 \pm 0.4$ & $0.4 \pm 0.7$ \\
\hline AT efficiency (\%) & $69.8 \pm 11.9$ & $74.3 \pm 13.9$ & $63.6 \pm 10.2$ & $72.8 \pm 12.3^{\alpha}$ & $67.6 \pm 15.0$ & $80.0 \pm 30.4$ & $65.6 \pm 14.9$ & $79.8 \pm 20.1^{\alpha}$ \\
\hline ST from play (n) & $11.9 \pm 3.5$ & $10.9 \pm 3.8$ & $9.0 \pm 3.2$ & $10.7 \pm 3.3$ & $5.8 \pm 2.5$ & $4.6 \pm 3.0$ & $4.7 \pm 2.5$ & $5.3 \pm 2.5$ \\
\hline ST from play (\%) & $77.1 \pm 14.0$ & $75.2 \pm 10.9$ & $72.5 \pm 12.8$ & $75.9 \pm 11.1$ & $74.9 \pm 16.1$ & $68.0 \pm 18.8$ & $68.6 \pm 20.4$ & $72.5 \pm 22.1$ \\
\hline ST dead ball (n) & $3.4 \pm 1.9$ & $3.3 \pm 1.4$ & $3.1 \pm 1.2$ & $3.3 \pm 1.6$ & $1.8 \pm 1.1$ & $1.9 \pm 1.1$ & $1.8 \pm 0.8$ & $1.9 \pm 1.5$ \\
\hline ST dead ball (\%) & $22.9 \pm 14.0$ & $24.8 \pm 10.9$ & $27.5 \pm 12.8$ & $24.1 \pm 11.1$ & $25.1 \pm 16.1$ & $32.0 \pm 18.8$ & $31.4 \pm 20.4$ & $27.5 \pm 22.1$ \\
\hline ST efficiency (\%) & $51.4 \pm 15.0$ & $55.3 \pm 15.2$ & $48.3 \pm 13.6$ & $43.7 \pm 15.1$ & $56.3 \pm 15.3$ & $62.3 \pm 24.1$ & $50.7 \pm 20.8$ & $38.6 \pm 18.4$ \\
\hline Total score & $8.8 \pm 3.1$ & $9.3 \pm 3.0$ & $6.4 \pm 2.4$ & $6.7 \pm 3.1$ & $4.7 \pm 2.1$ & $4.4 \pm 2.3$ & $3.5 \pm 1.6$ & $3.2 \pm 2.3$ \\
\hline No. of scores (n) & $7.8 \pm 2.4$ & $7.7 \pm 2.2$ & $5.9 \pm 2.1$ & $6.0 \pm 2.2$ & $4.2 \pm 1.6$ & $3.6 \pm 1.4$ & $3.3 \pm 1.6$ & $2.7 \pm 1.3$ \\
\hline Productivity & $2.4 \pm 0.8$ & $2.7 \pm 0.9$ & $1.8 \pm 0.8$ & $1.9 \pm 0.9$ & $2.4 \pm 1.0$ & $2.6 \pm 1.4$ & $1.9 \pm 1.1$ & $2.0 \pm 1.6$ \\
\hline Point (n) & $7.3 \pm 2.3$ & $6.8 \pm 2.1$ & $5.6 \pm 2.1$ & $5.7 \pm 2.0$ & $4.0 \pm 1.5$ & $3.3 \pm 1.3$ & $3.1 \pm 1.7$ & $2.4 \pm 1.0$ \\
\hline PT from play (n) & $4.8 \pm 1.9$ & $4.8 \pm 1.9$ & $3.6 \pm 2.0$ & $3.7 \pm 1.6$ & $2.5 \pm 1.4$ & $2.1 \pm 1.0$ & $2.0 \pm 1.6$ & $1.5 \pm 1.0$ \\
\hline PT dead ball (n) & $2.5 \pm 1.5$ & $2.0 \pm 1.0$ & $2.0 \pm 1.2$ & $2.0 \pm 1.6$ & $1.4 \pm 0.9$ & $1.2 \pm 0.8$ & $1.2 \pm 0.9$ & $1.0 \pm 1.0$ \\
\hline Goal (n) & $0.5 \pm 0.7$ & $0.8 \pm 0.8$ & $0.3 \pm 0.5$ & $0.3 \pm 0.6$ & $0.3 \pm 0.5$ & $0.4 \pm 0.6$ & $0.1 \pm 0.3$ & $0.3 \pm 0.6$ \\
\hline
\end{tabular}

$\mathrm{AT}=$ attack, $\mathrm{DF}=$ defense, $\mathrm{MF}=$ midfield, $\mathrm{ST}=$ shot, No. = number, av. = average, $\mathrm{PT}=$ point. Symbols indicate significantly different $(p \leq 0.05)$ from either first half or first quarter, using either a one sample t-test $(\alpha)$ or Wilcoxon signed rank test $(\beta)$.

Table 6. Summary of univariate comparisons for match halves and quarters, winners and losers - defensive play, mean \pm SD.

\begin{tabular}{|c|c|c|c|c|c|c|c|c|}
\hline \multirow{3}{*}{$\begin{array}{l}\text { Period } \\
24 \text { Games } \\
\text { Variable } \\
\end{array}$} & \multicolumn{4}{|l|}{ Half } & \multicolumn{4}{|l|}{ Quarter } \\
\hline & Winners & & Losers & & Winners & & Losers & \\
\hline & First & Second & First & Second & First & Fourth & First & Fourth \\
\hline Turnover (n) & $17.5 \pm 4.7$ & $15.0 \pm 4.8$ & $15.1 \pm 4.0$ & $13.5 \pm 3.7$ & $8.8 \pm 2.9$ & $6.6 \pm 2.9^{\alpha}$ & $8.0 \pm 2.6$ & $6.5 \pm 2.2$ \\
\hline TNR origin DF (n) & $9.1 \pm 2.4$ & $8.5 \pm 2.3$ & $9.3 \pm 3.5$ & $6.8 \pm 2.7^{\alpha}$ & $4.4 \pm 1.5$ & $3.9 \pm 1.6$ & $4.7 \pm 2.0$ & $2.9 \pm 1.6^{\alpha}$ \\
\hline TNR origin MF (n) & $7.5 \pm 3.4$ & $5.6 \pm 3.2^{\beta}$ & $5.3 \pm 2.6$ & $6.0 \pm 3.0$ & $4.0 \pm 2.3$ & $2.4 \pm 2.0^{\alpha}$ & $3.0 \pm 2.4$ & $3.2 \pm 1.9$ \\
\hline TNR origin AT (n) & $0.8 \pm 1.0$ & $1.0 \pm 1.2$ & $0.5 \pm 0.6$ & $0.8 \pm 1.0$ & $0.3 \pm 0.6$ & $0.3 \pm 0.4$ & $0.3 \pm 0.4$ & $0.4 \pm 0.8$ \\
\hline Tackle (n) & $46.0 \pm 13.6$ & $44.8 \pm 14.7$ & $47.5 \pm 12.7$ & $45.4 \pm 9.1$ & $23.9 \pm 7.7$ & $21.3 \pm 7.2$ & $24.5 \pm 6.2$ & $22.2 \pm 5.8$ \\
\hline TK success $(\%)$ & $12.4 \pm 4.2$ & $9.5 \pm 4.8^{\alpha}$ & $11.4 \pm 5.2$ & $10.3 \pm 4.8$ & $11.6 \pm 4.8$ & $9.7 \pm 7.2$ & $9.8 \pm 5.5$ & $11.2 \pm 4.5$ \\
\hline TK unsuccess. (n) & $40.4 \pm 12.6$ & $40.4 \pm 13.1$ & $42.3 \pm 12.5$ & $40.8 \pm 8.8$ & $21.1 \pm 6.8$ & $19.2 \pm 6.5$ & $22.1 \pm 5.9$ & $19.7 \pm 5.3$ \\
\hline TK unsuccess. (\%) & $87.6 \pm 4.2$ & $90.5 \pm 4.8^{\alpha}$ & $88.6 \pm 5.2$ & $89.7 \pm 4.8$ & $88.4 \pm 4.8$ & $90.3 \pm 7.2$ & $90.2 \pm 5.5$ & $88.8 \pm 4.5$ \\
\hline TK origin DF (n) & $17.5 \pm 6.6$ & $19.9 \pm 11.1$ & $20.4 \pm 7.3$ & $19.0 \pm 5.6$ & $9.0 \pm 4.7$ & $9.6 \pm 5.4$ & $10.6 \pm 4.1$ & $9.2 \pm 4.8$ \\
\hline TK origin MF (n) & $22.0 \pm 10.4$ & $19.6 \pm 8.5$ & $20.8 \pm 8.0$ & $18.5 \pm 6.9$ & $11.5 \pm 5.9$ & $9.5 \pm 4.3$ & $10.9 \pm 4.5$ & $8.8 \pm 4.1$ \\
\hline TK origin AT (n) & $6.5 \pm 4.5$ & $5.3 \pm 4.9$ & $6.2 \pm 3.7$ & $7.9 \pm 4.2$ & $3.4 \pm 2.8$ & $2.1 \pm 2.2^{\beta}$ & $3.0 \pm 2.2$ & $4.2 \pm 2.9$ \\
\hline Free kick won (n) & $9.9 \pm 4.0$ & $9.5 \pm 3.0$ & $8.8 \pm 3.9$ & $9.9 \pm 4.3$ & $5.0 \pm 2.4$ & $5.0 \pm 1.7$ & $4.4 \pm 2.4$ & $5.0 \pm 2.8$ \\
\hline FK origin MF (n) & $4.8 \pm 2.5$ & $4.2 \pm 2.1$ & $4.5 \pm 3.2$ & $5.2 \pm 3.0$ & $2.4 \pm 1.6$ & $2.1 \pm 1.3$ & $2.3 \pm 1.8$ & $2.6 \pm 1.8$ \\
\hline FK origin AF (n) & $2.9 \pm 1.7$ & $2.5 \pm 1.2$ & $2.5 \pm 1.5$ & $2.5 \pm 1.9$ & $1.6 \pm 1.1$ & $1.5 \pm 0.9$ & $1.3 \pm 1.0$ & $1.3 \pm 1.3$ \\
\hline Defensive actions*(n) & $72.4 \pm 14.7$ & $69.8 \pm 17.3$ & $72.3 \pm 15.9$ & $69.2 \pm 10.7$ & $37.1 \pm 9.0$ & $32.9 \pm 8.5$ & $37.4 \pm 7.9$ & $33.8 \pm 6.4^{\alpha}$ \\
\hline Def. actions DF (n) & $29.0 \pm 7.4$ & $30.9 \pm 11.9$ & $32.4 \pm 8.7$ & $28.6 \pm 6.8$ & $14.7 \pm 5.2$ & $14.9 \pm 5.8$ & $17.1 \pm 5.3$ & $13.8 \pm 5.2^{\alpha}$ \\
\hline
\end{tabular}




\begin{tabular}{|c|c|c|c|c|c|c|c|c|}
\hline Period & Half & & & & Quarter & & & \\
\hline 24 Games & Winners & & Losers & & Winners & & Losers & \\
\hline Variable & First & Second & First & Second & First & Fourth & First & Fourth \\
\hline Def. actions MF (n) & $34.1 \pm 11.3$ & $30.5 \pm 12.0$ & $31.0 \pm 11.0$ & $29.2 \pm 9.0$ & $17.8 \pm 6.8$ & $14.6 \pm 5.8$ & $16.3 \pm 5.4$ & $14.4 \pm 5.6$ \\
\hline Def. actions AT (n) & $9.3 \pm 5.2$ & $8.4 \pm 6.9$ & $8.8 \pm 4.4$ & $11.4 \pm 5.5$ & $4.6 \pm 3.2$ & $3.4 \pm 3.1$ & $4.0 \pm 2.7$ & $5.6 \pm 3.8$ \\
\hline Def. efficiency (\%) & $36.4 \pm 10.2$ & $27.2 \pm 12.4^{\alpha}$ & $30.2 \pm 11.9$ & $26.2 \pm 12.9$ & $34.4 \pm 14.9$ & $23.9 \pm 15.3^{\alpha}$ & $32.4 \pm 15.0$ & $28.0 \pm 22.8$ \\
\hline
\end{tabular}

$\mathrm{TNR}=$ turnover, $\mathrm{DF}=$ defense, $\mathrm{MF}=$ midfield, $\mathrm{AT}=$ attack, $\mathrm{TK}=$ tackle, $\mathrm{FK}=$ free kick, Def. $=$ defensive. $*$ Defensive actions include; combined turnovers, tackles and fouls committed (free kick conceded). Symbols indicate significantly different $(p \leq 0.05)$ from either first half or first quarter, using either a one sample t-test $(\alpha)$ or Wilcoxon signed rank test $(\beta)$.

\section{Discussion}

This is the first study to examine temporal changes in technical and tactical performance indicators in elite Gaelic football. Through examination of a sample of NFL and AIC games, significant differences between the first and second halves were evident in 10 variables ( 3 positive: 7 negative) in winning teams compared to 16 variables (4 positive: 12 negative) in losing teams. When the fourth quarter was compared to the first, significant differences were also found in 8 variables (negative) in winners and 23 variables (11 positive: 12 negative) in losers. The performance implications of these findings are discussed in relation to specific aspects of play incorporating, possession, passing, dead ball distribution, defense and offence, following consideration of variations in games statistics.

There were no significant difference in average playing times between the match halves and quarters. However, the significant decrease in ball in play time in both the second half and fourth quarter resulted in a significant increase in stoppage time during these periods. Unfortunately, the frequency, cause and duration of each stoppage was not recorded, making it impossible to determine the percentage of stoppage time due to injuries. As there was no difference in the number of free kicks conceded or dead ball restarts executed across match periods, it is likely that the increase in additional time is related in part to the rise in the number of substitutions made by both winners and losers and the relative increase in black cards issued in the second half of games. A similar reduction in ball in play time and increase in stoppage time was reported across halves in a retrospective analysis of FIFA World Cup final games between 1966 and 2010 [3]. The authors reported that an increase in the average duration and not frequency of stop events was found, which was important because the interaction between ball in play and stop periods can impact work to rest ratios and influence the intensity of subsequent play periods [3]. The ensuing trend towards shorter more intense periods of play [3], and increased high intensity running distance and actions [20], has the potential to impair physical performance, as fatigue has been suggested to increase towards the end of games [21]. Similarly, fatigue has been shown to impair activity profiles in Australian football through reductions in physical performance across halves and quarters [22, 23]. In the present study, it is unclear whether the increase in total stoppage time resulted in shorter, more intense periods of play in the second half. Nonetheless, the potential impact of physical decrements related to high intensity activities and fatigue on decision making and technical competence towards the latter stages of games cannot be discounted.

Gaining possession is an important facet of team sports, and possession of the ball was previously described as the most popular performance indicator in soccer [24]. Possession is required to initiate attacks and create scoring opportunities and in Australian football a significantly higher total time in possession was associated with winning quarters of games [25]. In the current study winners did not demonstrate any significant reduction in possession characteristics when the first half was compared to the second half, whereas losers experienced a significant decline in the total time collated for both team and player possessions, which suggests that the technical and tactical superiority of winners translated into more effective retention of possession. In addition, losers reported a significant decrease in the frequency of team possessions gained in both defense and midfield in the second half, which contributed to a significant reduction in successful transitions from defense to attack in this period.

The ability to counterattack, particularly from defense, but also from midfield, has previously been shown to distinguish winners from losers across full games [2], and this performance indicator is dependent on a team gaining and maintaining possession. Both winners and losers had a reduction in the frequency of team possessions in the fourth quarter. Among winners, this contributed to the significant decline in attacking frequency and in the number of player possessions in attack. Perhaps as a consequence, losers experienced significantly less player possessions in defense but also in midfield. Moreover, psychological factors that may impact a player's performance on a losing team need to be considered as researchers have recently suggested that if the outcome of a match is known in the second half due to superior opposition or a perceived unsurmountable lead, then player motivation may decline and potentially result in a decrease in work rate and reduced effort attempting to regain possession [26, 27].

It is plausible that the reduction in possession characteristics among losing teams was related to their passing profiles. In contrast to winners, losers had a significant reduction in the frequency and success rate of hand passes and in the number of kick passes executed across both comparison periods. These findings illustrate that winners consistently demonstrate superior technical execution and competence in passing compared to losers throughout the duration of a game. Interestingly, the number of unsuccessful kick passes recorded by losers decreased 
significantly in the fourth quarter. This small improvement in kick passing competence displayed by losing teams in the latter stages of games may be related to cognitive processes, for example enhanced decision making (i.e. choosing to kick pass when appropriate), or more proficient technical execution. Although, it is also reasonable to postulate that in comparison to the first quarter, less pressure was applied to either the (kick) passer or intended receiver due to a reduction in the number of players congested around the ball [3]. A reduction in player density provides players with more time and space [3], and this has previously been shown to enhance the probability of scoring in soccer when there was more than $1 \mathrm{~m}$ of free space around the player taking a shot [28]. It is likely that variations in player density result from changes in tactical strategy during situations when either the contest is still in dispute or the outcome of the game is likely known. Either way this metric is likely affected by levels of fitness and fatigue [3], and may decline particularly towards the latter stages of games when physical decrements in performance have been observed $[12,13,29]$ resulting in players having more space to pass or receive the ball.

Winners had a significant improvement in the percentage of free kick passes retained in the second half, whereas losers retained a significantly higher percentage of their own kick outs in the fourth quarter compared to the first. Although, these differences may have been caused by better technical execution of the dead ball pass, the influence of changes in the tactical deployment of players causing a lower player density for kick passer and/or receiver must be considered. In certain situations, teams may have withdrawn attacking players into defensive roles to increase the player density and defensive pressure on the ball carrier [3] within their defensive zone, while allowing the opposition to retain possession by not applying the same level of pressure outside of this zone. For example, teams that adopt a deep defensive screen often concede kick outs and/or free kicks to the opposition, to enable their own players to retreat into organized protective formations, which helps to explain the superior retention rate of short as opposed to long kick outs reported previously $[7,30]$.

The hypothesized lower player density may also be attributed to a reduction in the overall defensive intensity of winners in the latter stages of games. In support of this, winners had a significant decline in turnovers generated in midfield in the second half, perhaps related to a reduction in their overall tackling success rate. In addition, in the fourth quarter winners also obtained significantly less turnovers and performed significantly less tackles in attack. These findings combined with the significant decline in the number of attacks in both the second half and last quarter performed by winners, supports the contention that winners may have withdrawn some of their attacking players into defensive roles and therefore, may not have committed the same number of players to attack or to press the opposition in their own defensive zone. The tactic of withdrawing players into defensive roles to try and protect a lead, may not have been as effective as planned or anticipated as winners had a significant decline in defensive efficiency in both the second half and fourth quarter. This coincided with a significant increase in the attacking efficiency of losers, who executed a significantly higher number of shots in the second half, which may have been influenced by their ability to retain their own kick outs and translate the possession into scoring opportunities [7].

Conversely, there was a decline in the defensive performance of losers, as turnovers in defense were lower in both the second half and last quarter and, overall defensive actions and defensive actions in defense were significantly lower in the fourth quarter. The frequency of defensive actions in defense in losers may have been lower due to the opposition not committing as many players to attack and conversely losers releasing players from their defensive roles and committing more of these players to attack in order to obtain scores. As a consequence, in the second half losers performed more defensive actions in attack, albeit this finding was only approaching significance $(p=0.055)$. A combination of losing teams releasing players from their defensive roles and encouraging them to attack, and winning teams withdrawing some of their players into defensive roles, results in the attackers of winning teams experiencing a lower player density in the opposition's defensive area. This is obviously advantageous and preferable when attacking as this creates space and time for decision making and technical execution, particularly scoring attempts [3]. This hypothesized lower player density in the latter stages of games combined with the reduction in defensive performance reported in the second half in both winners and losers in this study, may partly explain the higher total scores often obtained by teams in the second halves of games.

A limitation of this study is that the performance profiles used complete match data and were therefore based on halves that had slightly different (although non-significant) durations, which also influenced the calculation of subsequent quarters. Future research could examine variables expressed relative to actual playing or ball in play times. In addition, the effect of final score line difference, i.e. $+3,6,9$, and 12 points on tactical and technical variables could be evaluated to determine the effect of winning and losing on performance. Moreover, the interaction of aggregated variables, established from a principal component analysis, with time and overall match outcome, could also be investigated. Finally, these technical and tactical profiles should be complimented with physical and psychological parameters to enable a holistic view of performance to be examined.

\section{Conclusion}

This study has highlighted temporal changes in technical and tactical variables across specific match periods that contribute to the performance of winners and losers and influence match outcome in elite Gaelic football competitions. Although decrements in the number of team possessions were observed in both winners and losers in the fourth quarter, additional declines in possession characteristics were more pronounced in losers, evinced by 
the reduction in the total time in both team and individual player possession from the first to the second half. Similarly, in contrast to winners, losers also demonstrated a significant reduction in the number of passes (hand and kick) performed across both halves and quarters. When both possession and passing characteristics are combined, the results suggest that winners do not experience the same temporal reduction in these aspects of technical performance compared to losers.

Although there was a reduction in the defensive efficiency of winners in the second half, losers were unable to translate their improved attacking efficiency, reflected in more shots executed in the second half, into a higher number of scores. This may be due to winners employing more effective defensive systems that resulted in losers attempting shots from outside the traditional scoring zone and/or because losers demonstrated inferior technical shooting competence, possibly as a consequence of fatigue and/or the impact of psychological factors (e.g. motivation). Nonetheless, the results indicate that defensive gaps appear in the latter stages of games, enabling scoring opportunities to be exploited, provided players have the fitness and tactical awareness to penetrate into the scoring zone and technical competence to convert shots into scores. In conclusion, these findings provide important insights relating to differences in the; possession, passing, dead ball distribution, offensive and defensive characteristics of winners and losers across match periods. This information can be used by coaches to assist in the development and implementation of their tactical performance strategies.

\section{Acknowledgements}

The authors would like to thank the players and teams who participated in the study.

\section{Disclosure Statement}

No potential conflict of interest was reported by the authors.

\section{Funding}

No external sources of funding were provided for this research

\section{References}

[1] Sport Ireland, 2018. Irish Sports Monitor Annual Report 2017.

[2] Gamble, D., J. Bradley, A. McCarren and N. M. Moyna, 2019. Team performance indicators which differentiate between winning and losing in elite Gaelic football. International Journal of Performance Analysis in Sport, 19 (4): 478-490.

[3] Wallace, J. L. and K. I. Norton, 2014. Evolution of World Cup soccer final games 1966-2010: Game structure, speed and play patterns. Journal of Science and Medicine in Sport, 17 (2): 223-228.
[4] Hughes, M. D. and R. M. Bartlett, 2002. The use of performance indicators in performance analysis. Journal of Sports Sciences, 20: 739-754.

[5] Reilly, T. and K. Collins, 2008. Science and the Gaelic sports: Gaelic football and hurling. European Journal of Sport Science, 8 (5): 231-240.

[6] Bradley, J. and P. O'Donoghue, 2011. Counterattacks in elite Gaelic football competition. International Journal of Performance Analysis in Sport, 11 (1): 159-170.

[7] Daly, D. and R. Donnelly, 2018. Data analytics in performance of kick-out distribution and effectiveness in senior championship football in Ireland. Journal of Sports Analytics, 4 (1): 15-30.

[8] Carroll, R., 2013. Team performance indicators in Gaelic football and opposition effects. International Journal of Performance Analysis in Sport, 13 (3): 703-715.

[9] McGahan, J. H., S. Mangan, K. Collins, C. Burns, T. Gabbett and C. O. Neill, 2018. Match-play running demands and technical performance among elite Gaelic footballers: Does divisional status count? Journal of Strength and Conditioning Research.

[10] Lynch, P. and R. Carroll, 2017. To compare the type of passing in Gaelic football at senior inter-county level historically to modern day. International Journal of Performance Analysis in Sport, 17 (6): 1-10.

[11] Allister, A., P. J. Byrne, C. D. Nulty and S. Jordan, 2018. Game-related statistics which discriminate elite senior Gaelic football teams according to game outcome and final score difference. International Journal of Performance Analysis, 18 (4): 622-632.

[12] Gamble, D., M. Spencer, A. McCarren and N. Moyna, 2019. Activity profile, PlayerLoad ${ }^{\mathrm{TM}}$ and heart rate response of Gaelic football players: A pilot study. Journal of Human Sport and Exercise, 14 (4): 1-15.

[13] Malone, S., B. Solan and K. Collins, 2017. The running performance profile of elite Gaelic football match-play. Journal of Strength and Conditioning Research, 31 (1): 30-36.

[14] Malone, S., B. Solan, K. D. Collins and D. A. Doran, 2016. Positional Match Running Performance in Elite Gaelic Football. Journal of Strength and Conditioning Research, 30 (8): 2292-2298.

[15] Rampinini, E., F. M. Impellizzeri, C. Castagna, A. J. Coutts and U. Wisløff, 2009. Technical performance during soccer matches of the Italian Serie A league: Effect of fatigue and competitive level. Journal of Science and Medicine in Sport, 12 (1): 227-233.

[16] Carling, C. and G. Dupont, 2011. Are declines in physical performance associated with a reduction in skill-related performance during professional soccer match-play? Journal of Sports Sciences, 29 (1): 63-71.

[17] Kempton, T., A. C. Sirotic, M. Cameron and A. J. Coutts, 2013. Match-related fatigue reduces physical and technical performance during elite rugby league match-play: A case study. Journal of Sports Sciences, 31 (16): 1770-1780.

[18] Mangan, S. and K. Collins, 2016. A rating system for Gaelic football teams: Factors that influence success. International Journal of Computer Science in Sport, 15 (2): 78-90. 
[19] Koo, T. K. and M. Y. Li, 2016. A guideline of selecting and reporting intraclass correlation coefficients for reliability research. Journal of Chiropractic Medicine, 15 (2): 155-163.

[20] Barnes, C., D. T. Archer, B. Hogg, M. Bush and P. S. Bradley, 2014. The evolution of physical and technical performance parameters in the english premier league. International Journal of Sports Medicine, 35 (13): 1095-1100.

[21] Waldron, M. and J. Highton, 2014. Fatigue and pacing in high-intensity intermittent team sport: An update. Sports Medicine, 44 (12): 1645-1658.

[22] Coutts, A. J., J. Quinn, J. Hocking, C. Castagna and E. Rampinini, 2010. Match running performance in elite Australian Rules Football. Journal of Science and Medicine in Sport, 13 (5): 543-548.

[23] Mooney, M., S. Cormack, B. O’Brien and A. J. Coutts, 2013. Do physical capacity and interchange rest periods influence match exercise-intensity profile in Australian football? International Journal of Sports Physiology and Performance, 8 (2): $165-172$.

[24] Lago, C. and R. Martín, 2007. Determinants of possession of the ball in soccer. Journal of Sports Sciences, 25 (9): 969-974.

[25] Gronow, D., B. Dawson, J. Heasman, B. Rogalski and P. Peeling, 2014. Team movement patterns with and without ball possession in Australian Football League players.
International Journal of Performance Analysis in Sport, 14 (3): 635-651.

[26] Redwood-Brown, A. J., C. A. Sunderland, A. M. Minniti and P. G. O'Donoghue, 2018. Perceptions of psychological momentum of elite soccer players. International Journal of Sport and Exercise Psychology, 16 (6): 590-606.

[27] Redwood-Brown, A. J., P. G. O'Donoghue, A. M. Nevill, C. Saward and C. Sunderland, 2019. Effects of playing position, pitch location, opposition ability and team ability on the technical performance of elite soccer players in different score line states. PLoS ONE, 14 (2): 1-21.

[28] Pollard, R., J. Ensum and S. Taylor, 2004. Estimating the probability of a shot resulting in a goal: The effects of distance, angle and space. International Journal of Soccer and Science, 2 (1): 50-55.

[29] Mohr, M., P. Krustrup and J. Bangsbo, 2003. Match performance of high-standard soccer players with special reference to development of fatigue. Journal of Sports Sciences, 21 (7): 519-528.

[30] Mangan, S., M. Ryan, S. Devenney, A. Shovlin, J. McGahan, S. Malone, C. O.' Neill, C. Burns and K. Collins, 2017. The relationship between technical performance indicators and running performance in elite Gaelic football. International Journal of Performance Analysis in Sport, 17 (5): 706-720. 